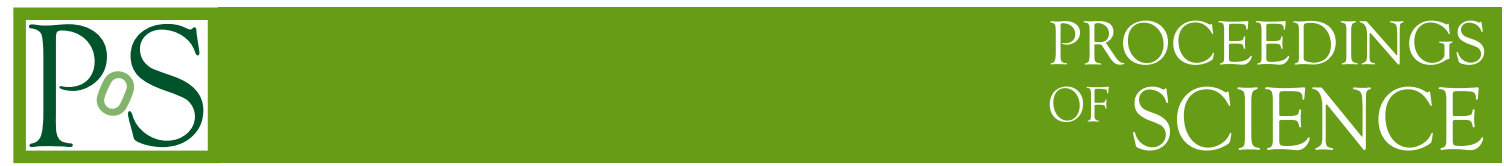

\title{
Tau reconstruction and identification at CMS
}

\section{Raman Khurana*}

On behalf of the CMS Collaboration

Saha Institue of Nuclear Physics

E-mail: raman.khurana@cern.ch

CMS has developed sophisticated $\tau$ identification algorithm for $\tau$ hadronic decay modes. Production of $\tau$ leptons decaying to hadrons is studied at $7 \& 8 \mathrm{TeV}$ centre of mass energy with 2011-2012 collision data collected by the CMS Detector. It is used to measure the performance of $\tau$ identification by measuring identification \& reconstruction efficiency and mis-identification rates from electrons, muons, and hadronic jets.

36th International Conference on High Energy Physics,

July 4-11, 2012

Melbourne, Australia

${ }^{*}$ Speaker. 


\section{Introduction}

Tau is the heaviest known lepton $\left(M_{\tau}=1.78 \mathrm{GeV} / \mathrm{c}^{2}\right)$ which decays into lighter leptons (BR $\sim 35 \%$ ) or hadrons $\tau_{h}$ (BR $\sim 65 \%$ ) in the presence of upto two neutrinos. CMS has developed Hadron Plus Strip (HPS) algorithm for reconstruction of hadronic tau decay modes. It uses decay mode identification techniques which allows to reconstruct $\tau_{h}$ with high efficiency and suppress the potentially large backgrounds from quarks and gluons that occasionally hadronize into low particle multiplicity jets.

\section{CMS tau reconstruction algorithm}

CMS utilises the fine granularity of the calorimeter, precision tracking and muon system to identify and reconstruct final state particles in an event. HPS [1] algorithm uses Particle Flow (PF) objects. In the PF approach information from all sub-detectors is combined to identify and reconstruct all particles, namely charged hadrons, photons, neutral hadrons, muons and electrons. The resulting list of particles is used to construct taus. The visible products of hadronic tau decays are used to reconstruct the taus. One of the major tasks in reconstructing the decay modes (one charged hadron along with upto two neutral hadrons and three charged hadrons) of the tau is determining the number of $\pi^{0}$ mesons produced in the decay. The HPS algorithm combines PF electromagnetic particles in strips, to take into account possible broadening of calorimeter depositions from photon conversions. The neutral objects are then combined with existing charged hadrons to reconstruct the hadronic tau. To differentiate $\tau_{h}$ from ordinary jets isolation criteria are used. The isolation requires that there are no charged hadrons or photons, apart from $\tau_{h}$ decay products, within an isolation cone around the $\tau_{h}$ direction. Three different working points are provided, loose, medium and tight, for different efficiencies and fake rates by adjusting the $E_{T}$ threshold for particles which are considered in isolation region. A final cross cleaning against Muons and Electrons is also applied.

This algorithm is being used with two different types of isolations :

- Cut based isolation

- Ring based isolation

The cut based isolation is calculated as the sum $p_{T}$ of the candidates in a solid cone of $\triangle \mathrm{R}=0.5$ around the reconstructed tau decay mode axis. MVA isolation uses the same PF candidates which are used for cut based isolation but for isolation calculation $p_{T}$ is summed in $\triangle \mathrm{R}$ rings around $\tau$ and Boosted Decision Tree (BDT) is trained against jet to $\tau$ mis-identification rate. MVA achieves lower mis-identification rate for same efficiency when compared with cut based isolation. Three working points are provided (loose, medium, tight) depending upon the threshold on isolation calculated in both the cases.

\section{Identification Efficiency}

The expected efficiency for the three working points is estimated using a sample of simulated $\mathrm{Z} \rightarrow \tau \tau$ events and is illustrated as a function of number of reconstructed vertices and $p_{T}$ in Figure 1. 
For the loose working point the efficiency is estimated to be approximately $60 \%$ for a wide range of transverse momentum.

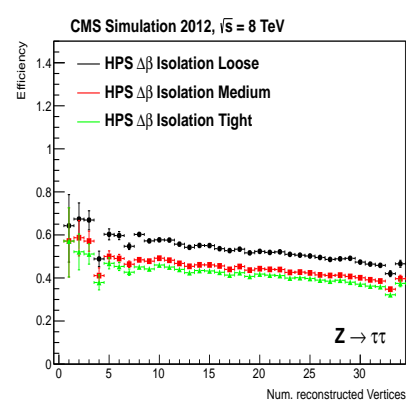

(a)

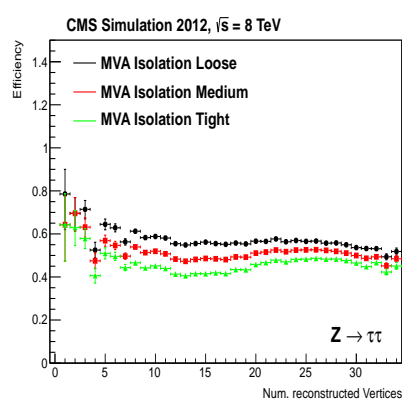

(b)

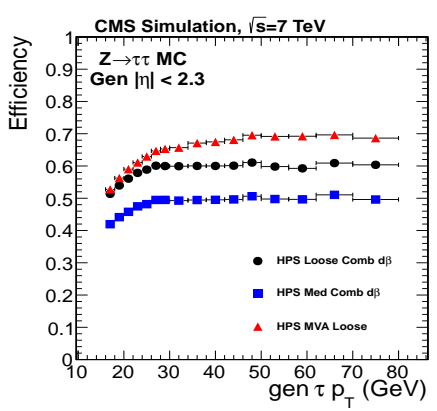

(c)

Figure 1: Tau isolation efficiency as a function of number of reconstructed vertices for loose, medium and tight working points for (a) cut based, (b) MVA isolation, (c) comparison of loose MVA isolation with loose, medium cut based isolation

\section{Measurement of mis-identification rate from jets}

The probability of the non-tau jets to be mis-identified as a $\tau$ candidate is called fake-rate and is estimated from data and is illustrated in Figure 2(a). The selection of QCD-type jets requires at least one jet with transverse momentum $p_{T}>15 \mathrm{GeV} / \mathrm{c}$ within $|\eta|<2.5$.

\section{Tau Energy scale}

Charged hadrons and photons are reconstructed with high precision using the Particle Flow techniques and hence the reconstructed $\tau_{h}$ energy is expected to be close to the true energy of its visible decay products. The quality of the $\tau_{h}$ energy scale has been examined by analyzing the $\mathrm{Z}$ $\rightarrow \tau \tau \rightarrow \mu \tau_{h}$ simulation events. Tau energy scale is found to be flat for all decay modes as shown in Figure 2(b)

\section{Physics Analysis using taus}

CMS has wide variety of physics analysis including Higgs searches and SUSY which uses HPS algorithm to reconstruct and identify the hadronic taus. Two such example of physics analysis using cut based and MVA isolation are described here to show performance of both the isolation techniques.

\section{1 $\mathrm{H} \rightarrow \mathrm{ZZ} \rightarrow \mathrm{l} \tau \tau$ analysis using Cut based isolation}

$\mathrm{H} \rightarrow \mathrm{ZZ} \rightarrow 11 \tau \tau$ uses cut based tau isolation to identify the hadronic taus. In this analysis Standard Model Higgs Boson decays to two $\mathrm{Z}$ bosons. One of the $\mathrm{Z}$ boson decays to pair of opposite signed leptons $\left(e^{+} e^{-}\right.$or $\left.\mu^{+} \mu^{-}\right)$and other $Z$ boson decays to pair of opposite signed taus, 


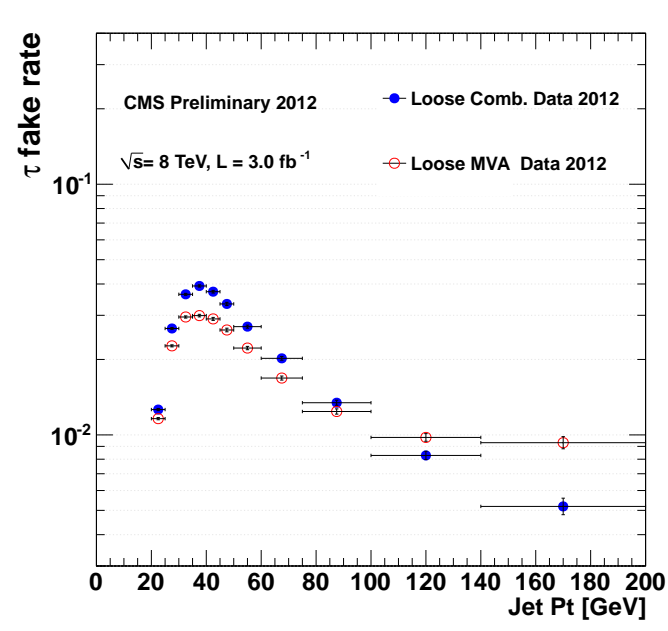

(a)

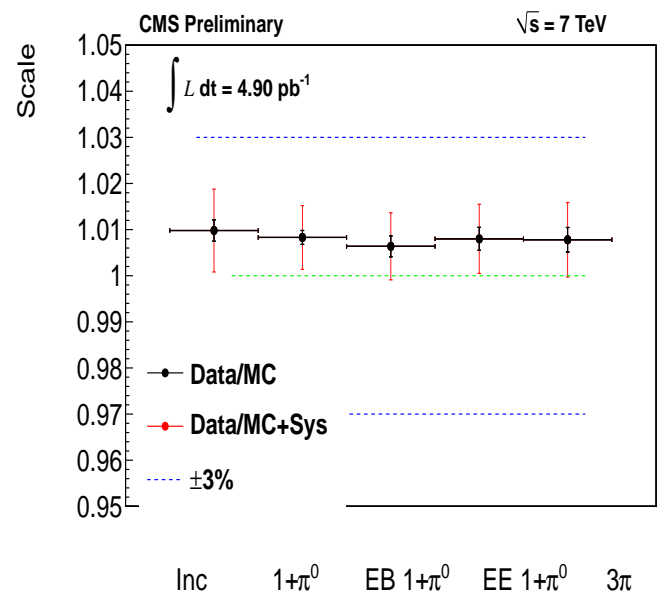

(b)

Figure 2: (a) Jet to tau mis-identification rate for loose MVA and cut based isolation, (b) tau energy scale for various decay modes.

which further decay to lighter leptons (electrons, muons) or hadrons. A total eight final states has been considered for this analysis when both the $\mathrm{Z}$ bosons are on shell which restrict the study in the high mass region $\left(M_{H}>180 \mathrm{GeV}\right)$. Figure 3 shows visible mass for $\mathrm{H} \rightarrow \mathrm{ZZ} \rightarrow 11 \tau \tau$ final states in data and simulation (left) and one of the event displays of $\mathrm{H} \rightarrow \mathrm{ZZ} \rightarrow 11 \tau \tau$ candidate events selected in data (right).

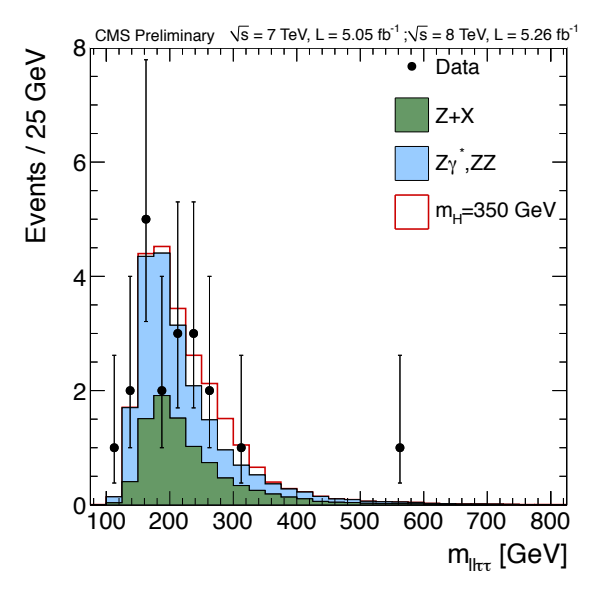

(a)

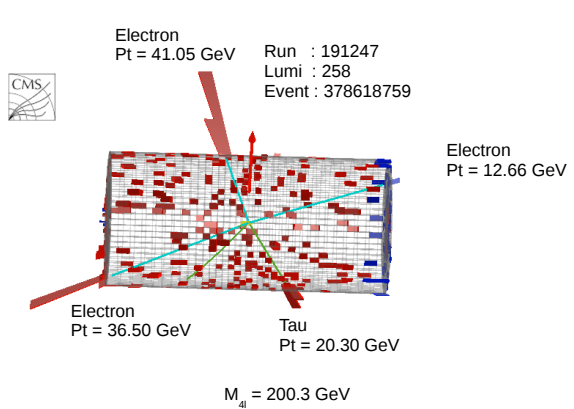

(b)

Figure 3: (a) $\mathrm{H} \rightarrow \mathrm{ZZ} \rightarrow 11 \tau \tau$ visible mass with all eight final states combined, (b) event display for one selected $e e \tau_{e} \tau_{h}$ candidate event

\section{2 $\mathbf{H} \rightarrow \tau \tau$ analysis using MVA isolation}

$\mathrm{H} \rightarrow \tau \tau$ uses MVA tau isolation to identify the hadronic taus. In this analysis those events are 
analysed where Standard Model Higgs Boson decay into a pair of oppositely charged taus, and taus further decay into lighter leptons (electrons or muons) and hadrons. Figure 4 shows $\tau \tau$ mass for $\mathrm{H}$ $\rightarrow \tau \tau \rightarrow \mathrm{e} \tau_{h}$ and $\mathrm{H} \rightarrow \tau \tau \rightarrow \mu \tau_{h}$ in data and simulation for VBF category.

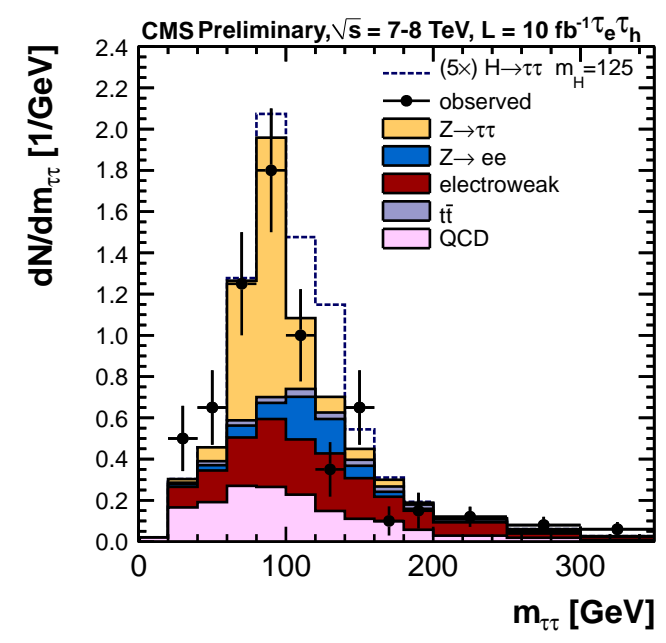

(a)

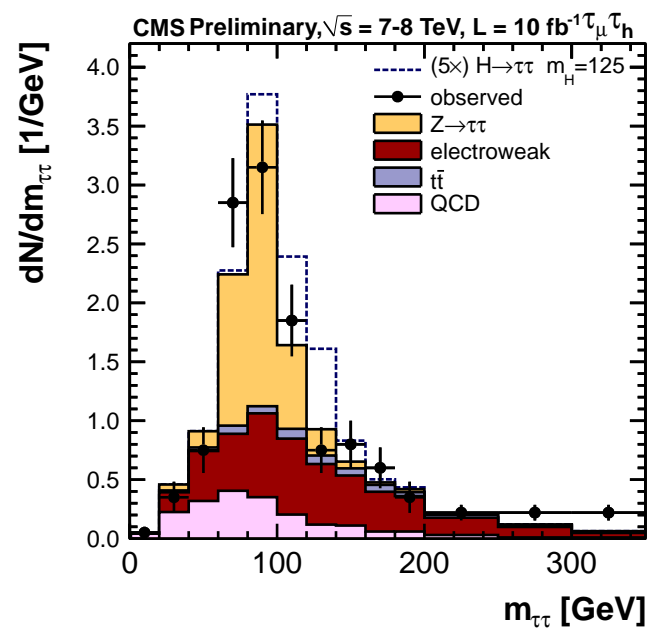

(b)

Figure 4: Visible mass distribution for (a) $\tau_{e} \tau_{h}$ and (b) $\tau_{\mu} \tau_{h}$ states for VBF category.

\section{Conclusions}

CMS has developed an advanced and robust $\tau$ reconstruction algorithm, which had been successfully commissioned with the data collected in 2011-2012. The efficiency of the HPS- $\tau$ algorithm is nearly $60 \%$ for a wide range of $\tau$ transverse momenta and with a few percents of fake-rate. The uncertainty of the $\tau$-identification efficiency was measured to be $6 \%$ and $\tau$ energy scale is of the order of $3 \%$.

\section{References}

[1] CMS Collaboration, Performance of tau-lepton reconstruction and identification in CMS, JINST 7 (2012) P01001, [ins-det/1109.6034].

[2] CMS Collaboration, Search for a Standard Model Higgs boson in the $H \rightarrow Z Z \rightarrow l l \tau \tau$ decay channel in pp collosion at $\sqrt{s}=7 \mathrm{TeV}, \mathrm{JHEP} \mathbf{0 3}$ (2012) 081.

[3] CMS Collaboration, Search for neutral Higgs bosons decaying to tau pairs in pp collisions, CMS PAS HIG-12-018.

[4] CMS Collaboration, Measurement of ZZ production cross section in $\mathrm{ZZ} \rightarrow 2 l 2 l^{\prime}$ decay channel in $p p$ collisions at $\sqrt{s}=8 \mathrm{TeV}$, CMS PAS SMP-12-014. 